\title{
Capacity-Fairness Trade-off Using Coordinated Multi-Cell Processing
}

\author{
Virgile Garcia ${ }^{1}$, Nikolai Lebedev ${ }^{1,2}$ and Jean-Marie Gorce ${ }^{1}$ \\ Contacts: virgile.garcia@insa-lyon.fr, lebedev@cpe.fr, jean-marie.gorce@insa-lyon.fr \\ ${ }^{1}$ University of Lyon, INRIA, INSA-Lyon, CITI, F-69621, Villeurbanne, France \\ ${ }^{2}$ CPE Lyon, BP 2077, F-69616, France
}

\begin{abstract}
Multi-cell processing, also called Coordinated Multiple Point (CoMP), is a promising distributed technique that uses neighbour cells' antennas. It is expected to be the part of next generation cellular standards such as LTE-A. Small cell networks in dense urban environments are limited by interferences and CoMP can strongly take advantage of this fact to improve cell-edge users' throughput. The present study introduces a distributed criterion for mobiles to select their optimal set of Base Stations (BS) to perform CoMP, and evaluates the impact of this association on the fairness and the total cell throughput. For that, we use a known theoretical expression for the capacity outage probability of CoMP under Rayleigh fading and evaluate the goodputs of antennas associations. The proposed criterion is used in combination with $\alpha$-fair resource allocation to perform a joint double-objective optimization of fairness and efficiency.
\end{abstract}

Keywords: Multicell processing, Capacity distribution, macro-diversity, interferences coordination, fairness, small cells, Rayleigh fading.

\section{INTRODUCTION}

Today, it is commonly anticipated that cellular networks will further evolve to denser and smaller cells, with a strong spatial frequency reuse, possibly with the whole spectrum resource made available within each cell (reuse 1) [1]. However, this increasing number of base stations (BS) with shorter coverage range critically increases the inter-cell interference level, especially at cell edges. Next generation (4G) systems intend to use small cells in urban environment to create local coverage to serve only few users at a time.

This dense and uncoordinated cells deployment makes the resource management a hard task, and previously used for $2 \mathrm{G} / 3 \mathrm{G}$ systems pattern-based resource allocation schemes turn out to be suboptimal, or too restrictive. More promising approaches for OFDMA systems rely on dynamic fractional frequency reuse [1], [2]. However, these approaches still suffer from different open problems including back-hauling overload, inter cells synchronization and signal coordination. A lot of work is devoted to reuse- 1 schemes and to the development of Self-Optimized and Self-Organized Networks (SON) [3] with minimal human involvement.

As a complementary solution, distributed MIMO in multicell environment appeared recently as a breakthrough technology to improve the energy-capacity trade-off of cellular networks [4]. CoMP was introduced, not for providing the huge increase of the total throughput [5], but rather to improve the SINR of cell edge users through spatial diversity. Edge- users will be able to decode a joint transmission from two or more BS in the same frequency band. That way, a better coverage is ensured with less users experiencing outage, and thus, a more intense reuse of the spectrum in dense areas is allowed. Several cooperation techniques of CoMP have been studied in [6], [7]. The maximal sum-rate capacity has been derived in [4] providing an upper-bound and revealing the potential of distributed MIMO.

The major issue related to these approaches is the need for coordination between cells: extensive data exchanges of channel state information (CSI) fed back by the mobiles, algorithms related parameters as well as synchronization data, making it impractical due to backhaul limitations. The evolved MIMO-like coordination, such as distributed beamforming or dirty-paper-coding requires a perfect time synchronisation in order to distributively generate in-phase signals or efficient precoding patterns, which require the knowledge of channel gains [6]. In [8], the authors evaluate the impact of a limited feedback. In [9], clustering methods are used to pre-define which BSs are working together.

We propose a distributed method with the reduced need of feedback by using less constrained techniques. As described below, the proposed method solely requires average users' SINR computed from powers received from the BS, and fed back by the mobile. The underlying technique is referred to as open-loop MIMO. In an optimal approach, the subband powers are weighted to control the interference level but it turns out that the interference levels cannot be predicted without a strict coordination between BS. This is why we study here a simplified resource allocation mechanism relying on constant transmission powers. Note that Wifi standard follows this principle. At a first glance, this assumption may appear strongly suboptimal, but the main advantage relies on interference stabilisation. On the other hand, adapting a station power to reduce interference to a given user has a limited effect due to the presence of numerous other interfering stations.

If it is easy to find work on multicell processing and clustering [9], [10], it is harder to find a practical criterion to decide on cooperation. In most cases, a predefined separation (based on distance or received signal strength) between inner and outer-users is considered, using Reuse1 or CoMP (e.g. [4]). Also, in most of works related to CoMP, the objective is formulated as a sum-rate capacity under power constraints, whereas the fairness is neglected. 
The novelties of this paper rely on: 1) A double-objective analysis of CoMP - to our knowledge, the problem of capacity-fairness trade-off has not been studied yet in the context of CoMP algorithms. 2) The proposal of a new distributed criterion for BS association, trading off fairness and throughput. This criterion may be used at mobile (or at its primary BS) to determine whether it requires other BS to cooperate. 3) A model that accounts for uncertainty due to fading, through our recent result on capacity outage distribution in CoMP with Rayleigh fading [11].

Section II details assumptions and system modelling. The proposed BS association procedure is presented. The distribution of the capacity under Rayleigh fading [11] is also derived to evaluate the resulting users' goodputs. In Section III, the resource allocation algorithm is provided, together with the fairness measure. In Section IV we describe a new cooperation criterion tunable according to different fairness constraints. We further evaluate the benefit of this approach for the efficiencyfairness trade-off, as illustrated in simulation results.

\section{SYSTEM MODEL}

The present study focuses only on downlink and is based on a dense small cell scenario, which typically corresponds to urban conditions with a high density of base-stations to ensure coverage continuity and user rate requirements. The whole system is considered as a snapshot and the time dimension is therefore omitted. The users are supposed to be randomly distributed on a 2D plan. We aim at optimizing the association of users to base station(s) and the resource allocation to users with different fairness objectives.

\section{A. Assumptions}

The proposed approach relies on the following rules and assumptions:

1) Each mobile is firstly associated with one BS referred to as the primary BS.

2) Similar to Wimax and LTE-A standards, a frame-based OFDMA modulation, with a set of time-frequency resource blocks (RB), is considered.

3) Each BS uses the whole RB set at maximal power for its users. Thus, the fairness level is managed by the policy defining the blocks allocated to each user.

4) Each RB experiences an independent Rayleigh fading.

5) Due to the previous assumptions, the interference level at a mobile user is almost stable within the block (averaged over the fading).

6) Each mobile feeds back a vector of average powers received from BSs in its vicinity.

7) We assume that any serving BS can request a neighbour BS to help serving one of its users; typically, this will be the strongest interfering BS for this user.

8) Since the exact and instanteneous CSI is not known, and no clock synchronisation between the BS assumed, the beamforming is not possible, and the Alamouti like precoding is used to maximize the mobile received SINR.

9) We assume single antenna receiver.

\section{B. Multi-cell coordination principle}

The BSs distributed coordination for the CoMP algorithm holds in four steps:

1) Power sensing: Each mobile measures the average power received from each neighbour $\mathrm{BS}$ during the beacon period. It returns a powers vector $P=\left[P_{1}, \cdots, P_{N}\right]$ to its primary BS.

2) CoMP capacity computation: Based on $P$, the BS computes the SINR of different CoMP associations, and selects the best policy. This procedure is detailed in next subsection.

3) Multiple BS association: According to the selected CoMP mode, a BS can request help to another BSs to simultaneously serve a given mobile. It is anticipated that even if only a small fraction of users adopts a CoMP approach, it can result in a capacity gain for all users.

4) RB blocks allocation: a negotiation phase is needed to coordinate the RB selection of CoMP users. BSs need to know the transmission rate and the set of cooperating stations used by each user. In this paper, for the sake of consistency, we don't address this algorithmic issue as we rather focus on expected performance. We thus assume that BSs always accept any cooperation request, and automatically adapt its resource allocation so that it respects neighbour's will and total available resources. The exact implementation issue and algorithmic performance will be addressed in a future work.

\section{SINR and downlink CoMP}

The combination of signals from two or more BSs allows users to improve their SINR level: the total interference power is decreased significantly by using the strongest interferer(s) as useful signal(s). $M$ users are considered in each cell. We note $\mathcal{N}_{i}$ the set of BSs that serve user $i$ in a coordinated manner, to create a distributed MISO link. At a given mobile $i$, on a given channel $l$, the received complex signal is:

$$
y_{i, l}=\sum_{n \in \mathcal{N}_{i}} h_{i, l, n} \sqrt{P_{i, n}} x_{i, l, n}+\sum_{n \notin \mathcal{N}_{i}} h_{i, l, n} \sqrt{P_{i, n}} x_{i, l, n}+z
$$

where $n$ is $\mathrm{BS}$ index, $z$ is the thermal noise, modeled as normal random variable with a $\sigma_{z}$ standard deviation $h_{i, l, n} \sim$ $\mathcal{C N}(0,1)$ are the fast fading random variables with Rayleigh distributed envelope; $x_{i, l, n}$ are the corresponding transmitted symbols; $P_{i, n}$ is the received power, averaged over the fast fading, including pathloss and shadowing effects from BS $n$ to mobile $i, P_{i, n}$ is supposed common to all considered frequencies. The shadowing is considered slowly varying with respect to the period of measurement reports from mobiles, with constant gain over the RB. We then suppose that $P_{i, n}$ is known at BS. Assuming a perfect CSI at the receiver side and coherent detection, the received SINR is:

$$
\gamma_{i, l}\left(\mathcal{N}_{i}\right)=\frac{\sum_{n \in \mathcal{N}_{i}}\left|h_{i, l, n}\right|^{2} P_{i, n}}{\sum_{n \notin \mathcal{N}_{i}}\left|h_{i, l, n}\right|^{2} P_{i, n}+\sigma_{z}^{2}},
$$

This SINR formulation results from the sum of random phase signals. In addition to the contribution of a (strong) interferer, 
CoMP offers a macro-diversity due to distant locations of the BSs. One of the objectives of this publication is to determine the set $\mathcal{N}_{i}$ that optimizes the system performances (spectral efficiency and fairness).

\section{Capacity outage probability}

To simplify notations, we drop the $i$ and $l$ indexes when no confusion is possible. According to Theorem 1 in [11], the probability for a channel of a given user with a set $\mathcal{N}$ of cooperating stations to be in capacity outage (i.e. that fails to reach a target capacity $R_{t h}=\log _{2}\left(1+\gamma_{t h}\right)$ ) is:

$$
\begin{aligned}
& P_{\mathcal{N}}^{\text {out }}\left(R_{t h}\right)=\mathbb{P}\left(\log _{2}(1+\gamma(\mathcal{N})) \leq R_{t h}\right)= \\
& 1-\sum_{n \in \mathcal{N}}\left(e^{-\frac{\gamma_{t h} \sigma_{z}^{2}}{P_{n}}} \prod_{j \in \mathcal{N}, j \neq n} \frac{P_{n}}{P_{n}-P_{j}} \prod_{k \notin \mathcal{N}} \frac{P_{n}}{P_{k} \gamma_{t h}+P_{n}}\right),
\end{aligned}
$$

\section{E. Parallel channels distribution}

LTE uses OFDMA, with sub-carriers grouped into resources blocks (RB) which form a time-frequency allocation slot. RB are assumed to experience a flat-fading across all sub-carriers they contain. Uncorrelated fading can be assumed between the RBs, which therefore can be considered as parallel channels, when allocated to the same user. Capacity outage probability of a given user with $L$ parallel channels [12] is:

$$
\begin{aligned}
& P_{\mathcal{N}, L}^{\text {out }}\left(R_{t h}\right)=\mathbb{P}\left(\frac{1}{L} \sum_{l=1}^{L} \log _{2}\left(1+\gamma_{l}\right)<R_{t h}\right) \\
& =\int \cdots \int_{\mathbb{R}^{L-1}} F_{\mathcal{N}}\left(L \cdot R_{t h}-\sum_{l=1}^{L-1} x_{l}\right) \prod_{l=1}^{L-1} f_{\mathcal{N}}\left(x_{l}\right) d x_{l}
\end{aligned}
$$

Where $f_{\mathcal{N}}\left(x_{l}\right)$ are the probability density functions (PDF) of the instanteneous capacity, obtained by derivation of (4). Considering a system without perfect CSIT, the best way to allocate $L$ resource blocks to a user is to spread the RBs all over the available spectrum (to obtain frequency diversity) with an equal power.

\section{F. Goodput}

We use the goodput as capacity metric $C(\mathrm{bps} / \mathrm{Hz})$. The goodput is defined as the user's spectral efficiency resulting from successful transmissions. For a given user $i$, once the set $\mathcal{N}_{i}$ is chosen and $L_{i}$ RBs allocated, his goodput $G_{i}$ can be maximized with respect to the transmission rate $R$.

$$
G_{i}=\max _{R} R\left(1-P_{\mathcal{N}_{i}, L_{i}}^{\text {out }}(R)\right)
$$

Independently of $\mathcal{N}_{i}, G_{i}$ grows with $L_{i}$, since parallel channels provide a frequency diversity and mitigate fading. But the allocation of the number of channels for each user depends on the required fairness among users and their respective SINR. This is introduced in Section III.

An optimization of the set $\mathcal{N}_{i}$ is proposed in Section IV taking into account the cost in resource of multiple BS transmission and depending on fairness criteria.

\section{CAPACITY-FAIRNESS TRADE-OFF}

To quantify the trade-off between fairness and efficiency, it appears necessary to introduce a double-objective characterisation of this system. As pointed out, the goodput of the user $i$ is the function of the amount of resources $L_{i}$ allocated to it, and of channels' SINRs $\gamma_{l}$ (via the capacity outage probability). Under the obvious constraint of limited total quantity of resources, this allocation need to be done fairly among users.

We choose to represent the Jain's index of fairness [13] and the cell total throughput (bps/Hz) as the two measures to balance on a per cell basis. A clear distinction has to be made between the $\alpha$-fairness allocation, which is the criterion for the resources sharing and the Jain's index, which is a measure of how fair this allocation is.

\section{A. Jain's index fairness}

The Jain's index $J \in[0,1]$, is defined as:

$$
J=\frac{\left(\sum_{i=1}^{M} L_{i} G_{i}\right)^{2}}{M \sum_{i=1}^{M}\left(L_{i} G_{i}\right)^{2}} .
$$

This index is advantageous by its fixed bounds and intuitive numerical interpretation (higher index means more fair): $J=$ $1 / M$ for the least fair allocation, meaning only the best user is served by the station. $J=1$ for the fairest case (i.e. all the users have the same capacity). Fairness can be computed either locally for each cell, or in a global way for the system.

\section{B. $\alpha$-fairness}

This study uses the well known $\alpha$-fairness [14] policy defining the resources partitioning between the users. $\alpha=0$ offers the maximum throughput, $\alpha=1$ is the proportional fairness, $\alpha=2$ is the harmonic fairness, $\alpha \rightarrow \infty$ is the maximum fairness (or "uniform capacity").

To be " $\alpha$-fair", an allocation $\mathbf{L}=\left[L_{1}, \ldots, L_{M}\right]$ of resources among $M$ users has to satisfy:

$$
\begin{cases}\arg \max _{\mathbf{L}} \sum_{i} \frac{1}{1-\alpha}\left(L_{i} C_{i}\right)^{1-\alpha} & \text { for } \alpha \geq 0, \alpha \neq 1 \\ \arg \max _{\mathbf{L}} \sum_{i} \log \left(L_{i} C_{i}\right) & \text { for } \alpha=1\end{cases}
$$

In the following, we use the $\alpha$-fair allocation of resources among users, since it allows a practical and easily parametrisable allocation method (through the adaptation of $\alpha$ ), straightforward for the desired tradeoff.

\section{COMP AND FAIRNESS}

\section{A. Cooperation criterion}

Mobiles are aware of their neighbour cells mean powers, so a practical algorithm based on these simple measurements should enable the system to select the cooperating BSs directly by the mobile or by the attached BS in a fully distributed manner. An appropriate criterion has to be defined to make this selection.

To fairly compare the performance of mobiles attached to one BS with those attached to several BSs, we need to take into account the multiplication of the resource cost induced by 
the latter: we need to look at users' per-BS spectral efficiency $S_{i}(\mathrm{bps} / \mathrm{Hz} / \mathrm{BS})$ :

$$
S_{i}=\frac{G_{i}}{\operatorname{card}\left(\mathcal{N}_{i}\right)}
$$

To determine the set of cooperating stations $\mathcal{N}_{i}$, every user then just need to maximize his $G_{i}$, with respect to $\mathcal{N}_{i}$. This intuitive utility function was used for example in [10], [15].

Figure 1 is an illustration of the trade-off that has to be made, using simulation environment described in subsection IV-B. Each point of the curves is taken for a particular value of $\alpha$ and its corresponding allocation $\mathbf{L}$, from which the total throughput is deduced. The arrows represent how the JainThroughput couples evolve using CoMP, for given values of $\alpha$ with criterion (10) applied at every mobile. This criterion, referred to as simple CoMP, can be seen advantageous when more fairness is required, compared to no CoMP case.

However, this somewhat arbitrary criterion used for uniform capacity (i.e., when the objective is to have the same throughput for every user) may not be optimal for other fairness policies: the performance in both efficiency and fairness depends on the BS association. One can see for instance that the gain in both fairness and efficiency is not the same for all fairness policies and using this simple coordination criterion is not worthy when looking at low fairness $(\alpha<1)$. This phenomenon is explained by the fact that low fairness tends to serve outer users with more resources than inner users. This decreases the cell efficiency for an increase of fairness.

We therefore propose in this paper another, more general criterion for users' association to BSs, in order to balance the impact of CoMP on the goodput and the number of cooperating stations to use. The proposed criterion has been motivated so that a simple parameter $\beta$ provides a large class of functions, including concave $(0 \leq \beta<1)$ and convex $(\beta>1)$ ones :

$$
\mathcal{N}_{i}=\arg \max _{\mathcal{N}} \frac{\left(G_{i}\right)^{\beta}}{\operatorname{card}(\mathcal{N})},
$$

Therefore, when $\beta$ is high, more users use cooperative antennas: a slight increase of the spectral efficiency using more BSs will easily compensate the cost in resources induced. On the contrary, with $\beta \rightarrow 0$, CoMP is never used, since the criterion judges any improvement of capacity as not significant enough.

Each different value of $\beta$ has its own impact on the network and associates differently BSs to users, and thus modifies the resource allocation result, regardless of the value of $\alpha$. This general formulation provides the criterion which can be adjusted jointly with the resources allocation to optimize the trade-off between capacity and fairness.

\section{B. Simulation results}

Simulation parameters can be found in Table I. A twodimensional area with $120 \mathrm{BSs}$ has been generated, but only 60 cells are taken into account to avoid border effects-the interference being the most important limiting effect, it is important not to limit the study to a very simplistic model (for example considering only first-tier interferers). The cells'

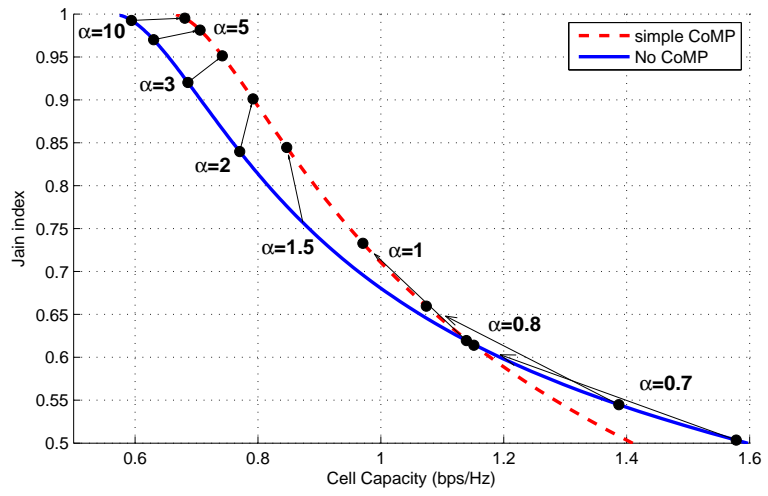

Fig. 1: Jain's index versus total throughput.

locations are randomly generated around the regular hexagonal grid. The users are uniformly distributed within each cell.

We are looking at the evolution of the couple EfficiencyFairness compared to Reuse1 mode. The objective is obviously to improve either the Jain's index or the total throughput, or even both when possible.

In the global system allocation (BS and resources), the parameters $\alpha$ and $\beta$ are not interdependent, as they are used to tune two separate parts of the optimization procedure. Nevertheless, as shown previously, they both have an impact on the performance and so it is interesting to investigate their joint effect. The objective is then to obtain the best possible couples $(\alpha, \beta)$ for the system.

Consider the Figure 2, The purple circles pattern shows the impact of $\beta$ for only one value of $\alpha=2$. The three curves represent no-CoMP (lower continuous blue line), simple CoMP (10) (black dashed line) and CoMP case with $\beta$ criterion (red dotted line) obtained as the envelope corresponding to the best couples $(\alpha, \beta)$, of the cloud of patterns representing all possible fairness-efficiency couples, obtained by extensive simulations. (In other words, the optimal couples are such combinations $(\alpha, \beta)$ that no other couple can improve both the fairness and total throughput).

We can see that the variation in both fairness and cell efficiency is hardly tractable, since it depends on the specific SINRs of users in each cell. However, it can be seen that when $\beta=0$, no user will ever choose multiple antennas, so that we will have the same result as for Reuse1. For growing values of $\beta$, CoMP first improves both fairness and efficiency before

\begin{tabular}{|c|c|}
\hline Parameter & Value \\
\hline Mean inter-cell distance & $200 \mathrm{~m}$ \\
Total transmit power $(P)$ & $30 \mathrm{dBm}$ \\
Path loss $(\mathrm{dB})$, d in meters & $34.53+38 \log _{10}(d)$ \\
Shadowing standard dev. & $10 \mathrm{~dB}$ \\
Thermal noise density & $-174 \mathrm{dBm} / \mathrm{Hz}$ \\
Fading $(h)$ & $\sim \mathcal{C N}(0,1)$ \\
Bandwidth & $20 \mathrm{MHz}$ \\
Number of UE/cell $(\mathrm{M})$ & 5 \\
\hline
\end{tabular}

TABLE I: Simulation parameters. 
reaching a limit and starts decreasing the efficiency, while still improving the fairness. When $\beta$ is too high, the performance of the system is even worse than without any BS cooperation, and we would rather use no coordination with a different $\alpha$ value instead.

Carrying out simulations for other values of $\alpha$, revealed the importance of the selected cooperation criterion value $\beta$, compared to the simple coordination criterion. When the fairness is important, the envelope tends to be similar to (10), but when lower fairness is required, an important gain in both metric appears, and keeps the CoMP more efficient and fair than without coordination.

Some other interesting questions arise. For example, can we describe among all the optimal couples, the interaction between the values of $\alpha$ and $\beta$. Specifically, one could ask whether for every $\alpha$-fair allocation, the corresponding optimal point is delivered by the same unique value of $\beta$ ? There is no simple parametrization rule and both $\alpha$ and $\beta$ may have several optimal points, again, depending on users' SINRs. We noted that the optimal value of $\beta$ tends to 1 when extreme fairness is required, but decreases with less fairness, tending to 0 for maximum throughput. This can be explained by the fact that low fairness criterion tends to give the most of resources to users with higher SINRs. Reallocating a part of good users' bandwidth to worse users strongly decreases their capacity, while only slightly increasing the low-rate users' throughput. The total throughput of the cell is then obviously reduced at a price of only slight increase of fairness.

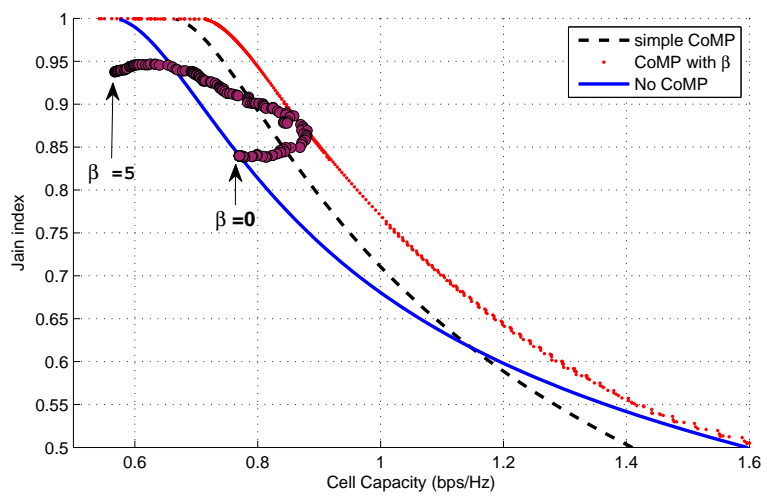

Fig. 2: Jain's index vs total throughput. Blue line for no cooperation, black dashed line is the simple CoMP, and the red dotted curve is the envelope of the best $(\alpha, \beta)$ couples. Purple points correspond to the $\alpha=2$, for $0 \leq \beta \leq 5$ case.

\section{CONClusion}

This paper introduces a generalized distributed criterion for mobiles to select their optimal set of Base Stations (BS) to perform the CoMP. We compared its performance with a simple CoMP scheme and no cooperation cases, and shown its benefit for most fairness-efficiency scenarios. We showed that our approach can improve both the fairness and the total throughput, especially for low SINR users which otherwise can consume too many resources.

\section{ACKNOWLEDGEMENT}

This work has been carried out in the frame of the joint lab between INRIA and Alcatel-Lucent Bell Labs on "Self Organizing Networks".

\section{REFERENCES}

[1] M. C. Necker. Towards frequency reuse 1 cellular FDM/TDM systems. In MSWiM '06, pages 338-346, New York, USA, 2006. ACM.

[2] A. L. Stolyar and H. Viswanathan. Self-organizing dynamic fractional frequency reuse in ofdma systems. In Proc. INFOCOM 2008. The 27th Conf. Computer Communications. IEEE, pages 691-699, 2008.

[3] 3GPP TR 36.902. Rel-9. E-UTRAN. Self-configuring and selfoptimizing network (SON) use cases and solutions., Dec. 2009.

[4] S. Jing, D. N. C. Tse, J. B. Soriaga, J. Hou, J. E. Smee, and R. Padovani. Multicell downlink capacity with coordinated processing. EURASIP Journal on Wireless Comm. Networks, 2008(5):1-19, 2008.

[5] Antti Toskala. "LTE advanced in 3GPP Release 10", keynote speach, COST2100, 9th MCM, Vienna, Austria, September 2009.

[6] E. Bjornson, R. Zakhour, D. Gesbert, and B. Ottersten. Distributed multicell and multiantenna precoding: Characterization and performance evaluation. In IEEE GLOBECOM 2009, pages 1-6, nov. 2009.

[7] J. Hoydis, M. Kobayashi, and M Debbah. On the optimal number of cooperative base stations in network MIMO systems. 2010. Available online: http://arxiv.org/abs/1003.0332.

[8] O. Simeone, O. Somekh, H. V. Poor, and S. Shamai. Downlink multicell processing with limited-backhaul capacity. EURASIP Journal on Advanced Signal Process, 2009:1-10, 2009.

[9] A. Papadogiannis, D. Gesbert, and E. Hardouin. A dynamic clustering approach in wireless networks with Multi-Cell cooperative processing. In IEEE International Conference on Communications ICC'08, pages 4033-4037, Beijing, China, May 2008.

[10] J. Zhang, R. Chen, J.G. Andrews, A. Ghosh, and R.W. Heath. Networked MIMO with clustered linear precoding. IEEE Trans. on Wireless Communications, 8(4):1910-1921, 2009.

[11] V. Garcia, J.-M. Gorce, and N. Lebedev. Outage Probability for MultiCell Processing under Rayleigh Fading. Accepted, IEEE Communications Letters, available online: http://hal.archives-ouvertes.fr/inria00519390/en/.

[12] D. Tse and P. Viswanath. Fundamentals of wireless communication Cambridge University Press, New York, NY, USA, 2005.

[13] R. Jain, D. Chiu, and W. Hawe. A quantitative measure of fairness and discrimination for resource allocation in shared computer systems. Technical Report DEC-TR-301, Digital Equipment Corporation, Sept. 1984.

[14] J. Mo and J. Walrand. Fair end-to-end window-based congestion control. IEEE/ACM Trans. Netw., 8(5):556-567, 2000.

[15] V. Garcia, N. Lebedev, and J-M. Gorce. Multi-cell processing for uniform capacity improvement in full spectral reuse system. In COGnitive systems with Interactive Sensors, Paris, France, Nov. 2009. SEE, IET. 